\title{
NEXT Ion Optics Modeling of Total Thruster Performance
}

\author{
Jerold W. Emhoff* and Iain D. Boyd $\mathrm{d}^{\dagger}$ \\ University of Michigan, Ann Arbor, Michigan, 48109
}

\begin{abstract}
An axisymmetric ion optics model is applied to the NEXT ion engine. The model is used to simulate the performance of the entire thruster by modeling several apertures at varying radii on the thruster face and integrating the results. The integrated results are compared to experimentally measured data for the NEXT thruster, showing good agreement in most areas. The primary area of discrepancy is in the accelerator grid current, although erosion results suggest that the measured current is unaccountably high. The model is also used to estimate the life of the thruster before the onset of electron backstreaming. Three separate methods are applied, and each predicts thruster failure after approximately 40,000 hours of operation, or $845 \mathrm{~kg}$ of xenon throughput.
\end{abstract}

\section{Introduction}

$\mathrm{T}$ HE NEXT ion engine is the successor to the very successful NSTAR ion engine. ${ }^{1}$ NEXT, or NASA's Evolutionary Xenon Thruster, has a $40 \mathrm{~cm}$ beam diameter, compared to NSTAR's $30 \mathrm{~cm}$ diameter. The thruster also has an improved discharge chamber design, resulting in a flatter current density profile across the thruster face. ${ }^{2}$ NEXT has undergone a 2000 hour wear test, from which experimental results for performance and thruster life have been obtained. ${ }^{3,4}$

Simulation of ion optics to date have focused primarily on accurately modeling the performance of the ion optics and the life of the thruster. Several 3-D models are currently in development: at NASA Glenn Research Center, ${ }^{5}$ Colorado State University, ${ }^{6}$ Virginia Tech, ${ }^{7}$ and by Okawa et al. ${ }^{8}$ The use of a full 3-D model allows accurate simulation of erosion on the downstream face of the accelerator grid as well as better comparison to experimental measurements. The model presented in this work is an axisymmetric simulation of a single ion optics aperture. This model has been under development for several years, ${ }^{9}$ and has previously been applied to both the NSTAR ${ }^{10}$ and NEXT ${ }^{11-13}$ ion engines. The simulation is applied to NEXT in this work, with the goal of comparing simulated performance and erosion to the experimentally measured data. This is accomplished by simulating several apertures across the thruster face and integrating the results to obtain performance for the entire thruster. This type of analysis has not previously been conducted in ion optics simulations. Three methods are also used to estimate the life of the thruster before failure due to electron backstreaming. This provides new insight into what type of erosion simulation is required in order to obtain an accurate estimate of the thruster life.

The paper first briefly discusses the model operation and operating conditions. Next, the methodology and results for total thruster simulation are given, including a discussion of the integration procedure, comparison to experimental data, and discussion of the accelerator grid current. A brief study of electron backstreaming is given, followed by simulation results for thruster life.

\section{Model Operation}

The computational model simulates a single 2-D axisymmetric aperture in an ion thruster. A computational mesh composed of evenly spaced rectangular cells is used to track particles in the simulation. The optics of the thruster are simulated using boundary cells in the domain. These cells may be arranged in an irregular way, allowing the simulation of cusps on the barrels of the grids. Figure 1 shows a typical domain,

*Research Scientist, Aerospace Engineering, 2012 FXB, AIAA Member

${ }^{\dagger}$ Professor, Aerospace Engineering, 3012 FXB, AIAA Associate Fellow 


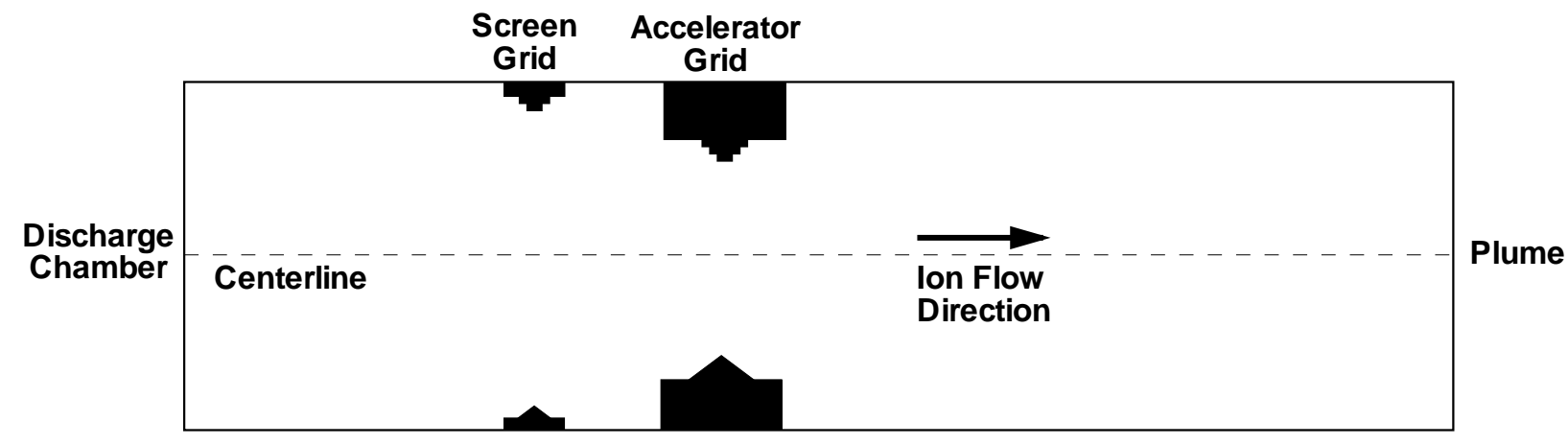

Figure 1. Plot of the simulation domain. The upper half is the meshed simulation domain, while the bottom half is the actual domain geometry.

with the top half of the plot showing the meshed representation, and the bottom half showing the actual geometry. The radius of the domain is set to half the center-to-center spacing between adjacent apertures in the ion optics.

The code uses the Particle-In-Cell ${ }^{14}$ (PIC) method to simulate xenon ions, xenon neutrals, and doubly charged xenon ions. Each computational particle has a numerical weight that indicates the actual number of atoms represented by the particle. Flow field quantities for each cell are obtained by averaging the properties of all the particles in the cell, taking into account the weight of each particle. The potential field accelerates ions self-consistently and electrons are modeled as a fluid via the Boltzmann relation. The direct simulation Monte Carlo (DSMC) method ${ }^{15}$ is used for processing particle collisions. Both charge exchange (CEX) and momentum exchange collision types are simulated. On the order of 100,000 iterations are performed to reach a steady-state of ion flow, after which data are sampled for another 100,000 iterations.

The sputter yield for xenon impacting on molybdenum is determined in the simulation by two models. The first model is a curve fit to experimental data giving the energy dependence of the sputter yield ${ }^{16}$ for impacts normal to the surface. The second model gives the relative yield as a function of the angle of impact for $300 \mathrm{eV}$ particles. ${ }^{17}$ The calculated results from these two models are multiplied to obtain the total sputter yield for a given impact.

The conditions simulated for the NEXT thruster are set to the maximum power operating point of the thruster, and the beamlet on the thruster centerline is usually modeled. This involves a discharge potential of $1800 \mathrm{~V}$, a screen grid potential of $1776 \mathrm{~V}$, and an accelerator grid potential of -210 V. The plume plasma potential is set to $22 \mathrm{~V}$. The beamlet current for the centerline aperture is approximately $0.168 \mathrm{~mA}$.

\section{Total Thruster Simulation}

Performance data has been measured for the NEXT ion engine during a 2000 hour wear test. ${ }^{18}$ In order to obtain accurate computational results for comparison to this performance data, it may be necessary to simulate several apertures at varying radii on the thruster optics. The results from these simulations can then be integrated to give results for the entire thruster. However, the ion current determines much of the performance of the thruster, so it may be possible to estimate the thruster performance based on a single simulation. The results from that simulation can be scaled appropriately by the beamlet current to give results for all apertures.

\section{A. Beam Current Density Profile Scaling}

A beam current density profile ${ }^{3}$ for the NEXT ion engine operating at $3.52 \mathrm{~A}$ is plotted in Figure 2. This profile can be used to determine the radius of a given single-aperture simulation by matching the beamlet current density of the simulation to the profile. It can also be used to integrate thruster performance based on a single simulation. However, the experimental current density profile presents two problems. First, the profile extends beyond the beam extraction area of the thruster. This is resolved by scaling the radius by a factor of approximately 0.988 at each point, such that the second-to-last point on the profile is at $\pm 20 \mathrm{~cm}$. The last point is ignored completely. 


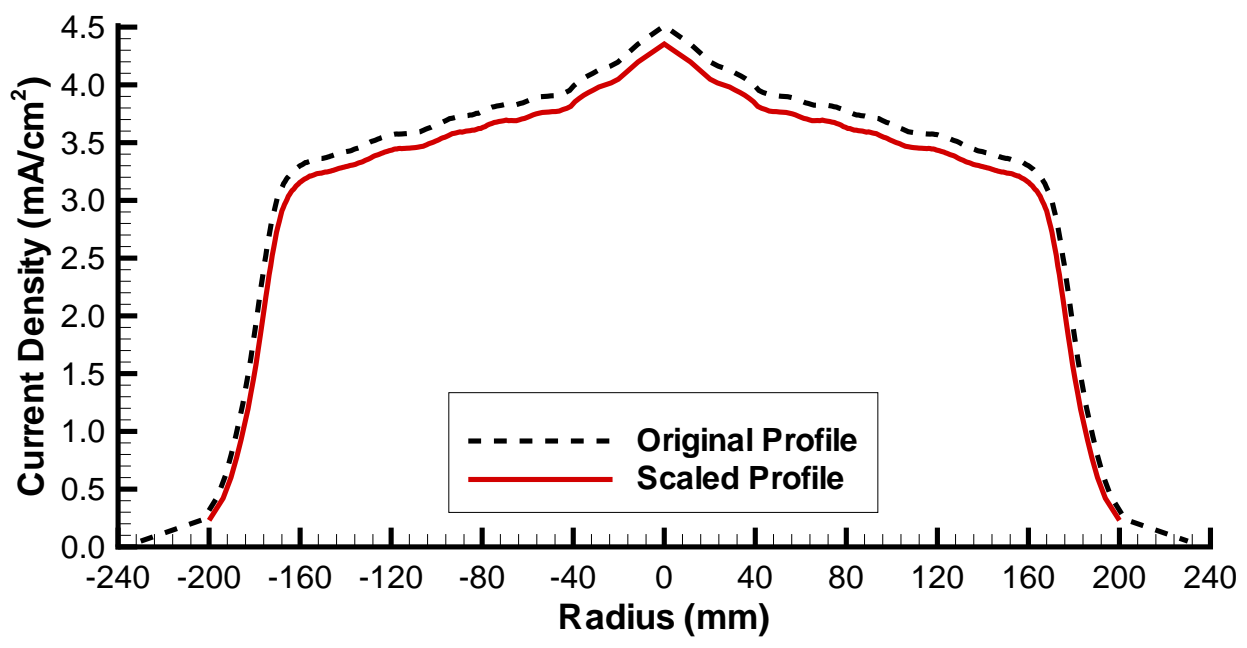

Figure 2. Experimentally measured and scaled beam current density profiles for the NEXT ion engine extracting 3.52 A of beam current. The scaled profile ignores the outer-most experimental point, then scales the radius of the remaining points to be within $\pm 200 \mathrm{~mm}$. The radius-scaled profile is then scaled such that the integral of the profile gives the correct beam current.

Second, when integrated, the profile gives a higher beam current than the thruster is operating at. This is accounted for by scaling the profile down such that integrating it gives the correct beam current. This scaling factor is about 0.965 at the $3.52 \mathrm{~A}$ beam current operating point. The profile resulting from these two scaling methods has a flatness parameter of about 0.71 , the same as is measured experimentally. This indicates that the scaled profile is reasonably accurate for the purposes of locating simulated apertures on the thruster. The scaled version of the profile is also shown in Figure 2.

\section{B. Multiple-Aperture Simulation Results}

The multiple-aperture simulation results use Dalgarno ${ }^{19}$ momentum-exchange collision cross-sections with isotropic scattering and varying aperture diameters. The ion optics used in the NEXT 2000 hour wear test have decreasing aperture diameters as the thruster radius increases, due to the grid manufacturing process. In order to obtain accurate results, the aperture diameters are varied in the simulations as well.

In each simulation, the upstream domain length is set to $2 \mathrm{~mm}$ for all but the lowest current case. For this case, the domain length is set to $4 \mathrm{~mm}$. The downstream domain length is $4 \mathrm{~cm}$ in each case. Sputtered grid material is not modeled in any of the simulations.

Figure 3 shows the variation of both grid aperture diameters as a function of radius, normalized to the nominal centerline aperture diameter. ${ }^{3}$ Also shown are the simulated aperture locations and diameters. The experimental grid diameters were measured using a pin gauge, thus the aperture diameters may be up to 0.001 inch larger in reality. The simulations use a constant radial cell size, so there is some error in the representation of the aperture diameters and cusp structures. Also, when a simulation is run, the exact output beamlet current is not known beforehand, so an estimate of the radius on the thruster is required in order to determine what the aperture diameters will be. However, the aperture diameters change very rapidly for mid-range thruster radii, and the beamlet current changes very slowly. The result is that a small change in beamlet current may produce a large change in aperture diameter. This explains the discrepancies seen in the plot.

The computed thrust, beamlet current, mass flow rate, and accelerator grid current are shown in Figure 4 as a function of radius. All quantities are normalized to the centerline value. The thrust varies exactly linearly with the beamlet current, as the beam ions produce almost all of the thrust. The mass flow rate is nearly linear with the beamlet current, but at low currents the mass flow rate does not drop as quickly as the beamlet current. This is because the discharge neutral density is held constant across all apertures, so the total flow rate does not drop as quickly when the ion flow rate is small.

The accelerator grid current decreases more quickly as the radius increases than the beamlet current or thrust. This is caused by the decrease in screen and accelerator grid aperture diameters. As the diameter 


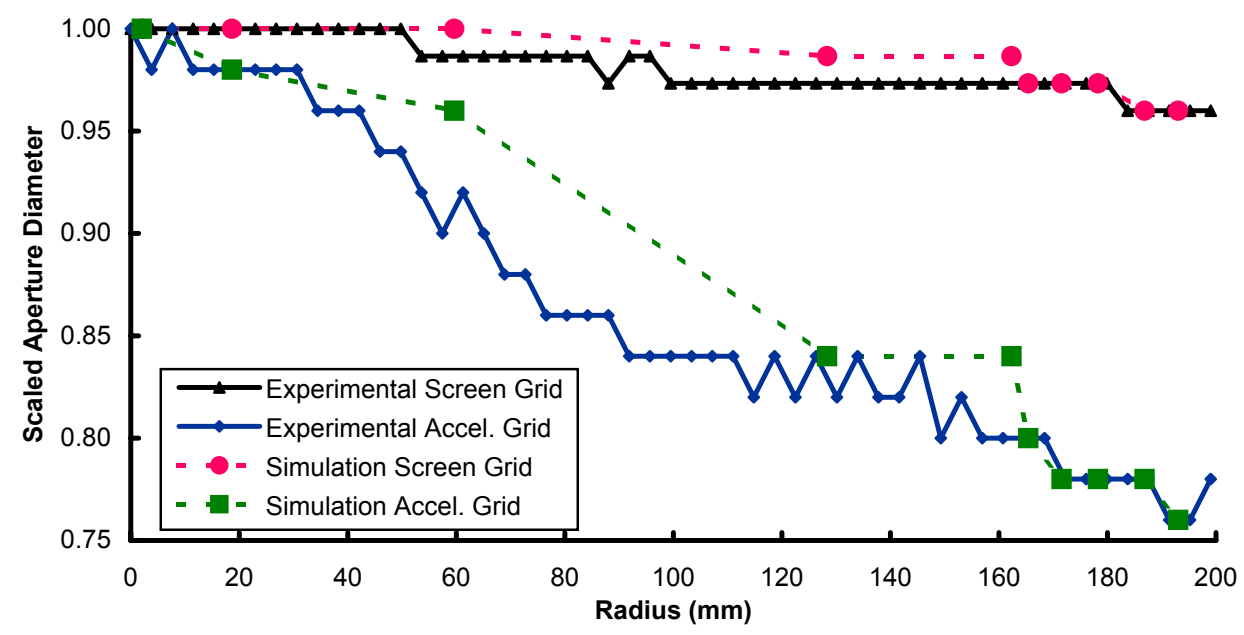

Figure 3. Variation of the accelerator and screen grid aperture diameters as a function of radius on the thruster. The simulated aperture diameters are also shown.

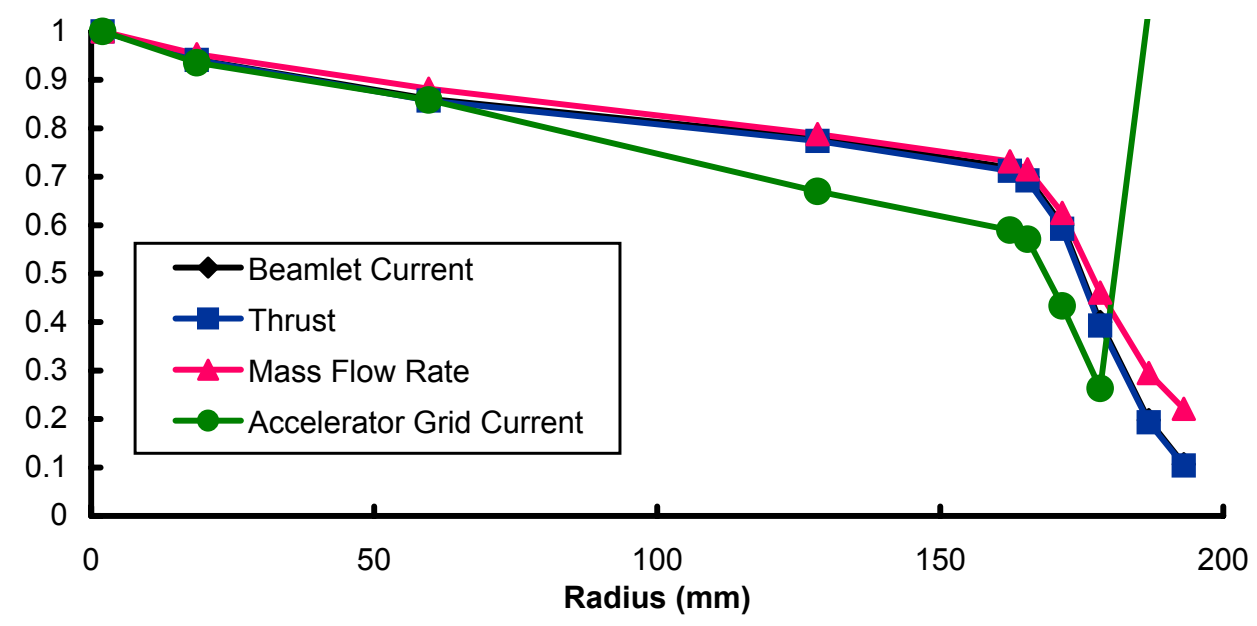

Figure 4. Variation of simulated performance quantities as a function of radius. 
decreases, fewer neutrals from the discharge chamber are able to flow through the optics, leading to a lower neutral density downstream of the ion optics. The CEX production rate is a linear function of both ion and neutral density, so as the neutral density decreases, the accelerator grid current will decrease as well. Also, the two simulated apertures at the highest radii have much larger accelerator grid currents than the other apertures. The low beamlet current in both cases induces crossover and direct impingement of beam ions on the accelerator grid barrel. This direct impingement will in reality quickly erode the aperture wall until the beamlet no longer impinges directly.

\section{Integration Procedure}

The multiple-aperture simulation results are integrated to obtain total thruster performance results. Each relevant quantity obtained for individual apertures is divided by the simulation area in order to obtain a density value instead. The density value is then assumed to vary linearly between the simulated apertures. The resulting linear approximating function is integrated between the points and over the thruster surface area to give the contribution between the points. The individual contributions are then summed over all points to obtain the total thruster performance quantities. In equation form, the process is as follows for a quantity $Q$ :

$$
\begin{aligned}
q_{i} & =Q_{i} / A_{\text {sim }} \\
a_{i} & =\frac{q_{i}-q_{i+1}}{r_{i}-r_{i+1}} \\
b_{i} & =-a_{i} r_{i}+q_{i} \\
Q_{\text {Total }} & =\pi \sum_{i=1}^{N-1} \frac{2 a_{i}}{3}\left(r_{i+1}^{3}-r_{i}^{3}\right)+b_{i}\left(r_{i+1}^{2}-r_{i}^{2}\right) .
\end{aligned}
$$

Here the $a_{i}$ and $b_{i}$ are the slopes and intercepts of the linear approximation functions, respectively, $A_{s i m}$ is the simulated aperture domain area, and $N$ is the number of simulated points.

To approximate the total thruster performance using a single aperture, the quantities of interest are scaled according to the beam current density profile in order to obtain values at all points. The scaled values are then integrated in the same way as above in order to obtain total thruster performance results. This procedure will give the best results when the quantity of interest varies linearly with the beamlet current. The centerline beamlet current simulation is used in these cases as the base, but any simulation can be used. The values from the simulation are simply scaled up or down depending on the simulated aperture's position on the current density profile.

\section{Total Thruster Performance Results}

In Table 1, integrated simulation performance results are compared to experimental data. Both multipleaperture and single-aperture simulation performance results are shown. As the table shows, the multiple aperture integration gives excellent comparison to the experimental results in most cases. The thrust is nearly exact, the mass flow rate is only slightly high, and the specific impulse is also very close to the experimental value. The mass flow rate is higher due to a slightly high neutral flow rate imposed in the simulations. Note that the experimental thrust is actually a calculated value, as the thrust is not measured directly.

The screen grid current is higher than the experimental value by only a small amount. The difference seen here may be caused by the inaccuracy of the simulation of the cusp structure on the screen grid aperture wall. Also, the accelerator grid current is about 3 times smaller than the experimental data. The beam current should be very close to the experimental value, as the experimental current density profile is used to determine the aperture radii. Thus, any error in the beam current integration will induce integration error for all quantities. Also, the beam current for the single-simulation case should be exact, as the beam current density curve is simply integrated to obtain the value.

The single-aperture integration results do not compare as well as the multiple aperture results. This is expected, especially given that quantities such as mass flow rate and accelerator grid current do not vary linearly with the beamlet current. However, there is still excellent prediction of thrust and screen grid current. There is some difference from the multiple-aperture results in accelerator grid current and erosion rate, much of which is due to the direct impingement seen in the outer, low current apertures. 
Table 1. Comparison of performance quantities for multiple aperture simulations and single aperture integration against experimental data from the NEXT ion engine 2000 hour wear test. ${ }^{3}$

\begin{tabular}{|r|c|c|c|}
\hline & $\begin{array}{c}\text { Experimental } \\
\text { Data }^{3}\end{array}$ & $\begin{array}{c}\text { Multiple } \\
\text { Simulation }\end{array}$ & $\begin{array}{c}\text { Single } \\
\text { Simulation }\end{array}$ \\
\hline Mass Flow Rate (mg/s) & 5.87 & 6.01 & 6.24 \\
Thrust (mN) & 237 & 239 & 241 \\
Specific Impulse (s) & 4117 & 4055 & 4001 \\
Beam Current (A) & 3.52 & 3.51 & 3.52 \\
Screen Grid Current (A) & 0.44 & 0.465 & 0.462 \\
Accelerator Grid Current (mA) & 12.5 & 4.77 & 3.88 \\
Erosion Rate (mg/hr) & - & 26.46 & 5.64 \\
\hline
\end{tabular}

It should be noted that the simulated screen grid currents listed in Table 1 are corrected values. The current actually simulated by the model is approximately $0.13 \mathrm{~A}$ in each case. However, the axisymmetry of the simulation does not account for the hexagonal arrangement of the thruster apertures. The screen grid current is corrected for ions collected on the areas of the screen grid which are not simulated. The correction to the current is about twice as large as the simulated amount of current. In Table 1, approximately $30 \%$ of the current is simulated, while the corrected current provides the remaining $70 \%$.

\section{E. Accelerator Grid Current Discussion}

The property offering the poorest comparison between simulation and experiment, for both multiple and single aperture integration, is the accelerator grid current. In both cases, the simulated accelerator grid current is low by a factor of about 3. The deficit in current should be seen in the erosion rate as well, as both depend on CEX ions. If the simulated erosion rate is three times too low as well, then the simulation is clearly operating incorrectly.

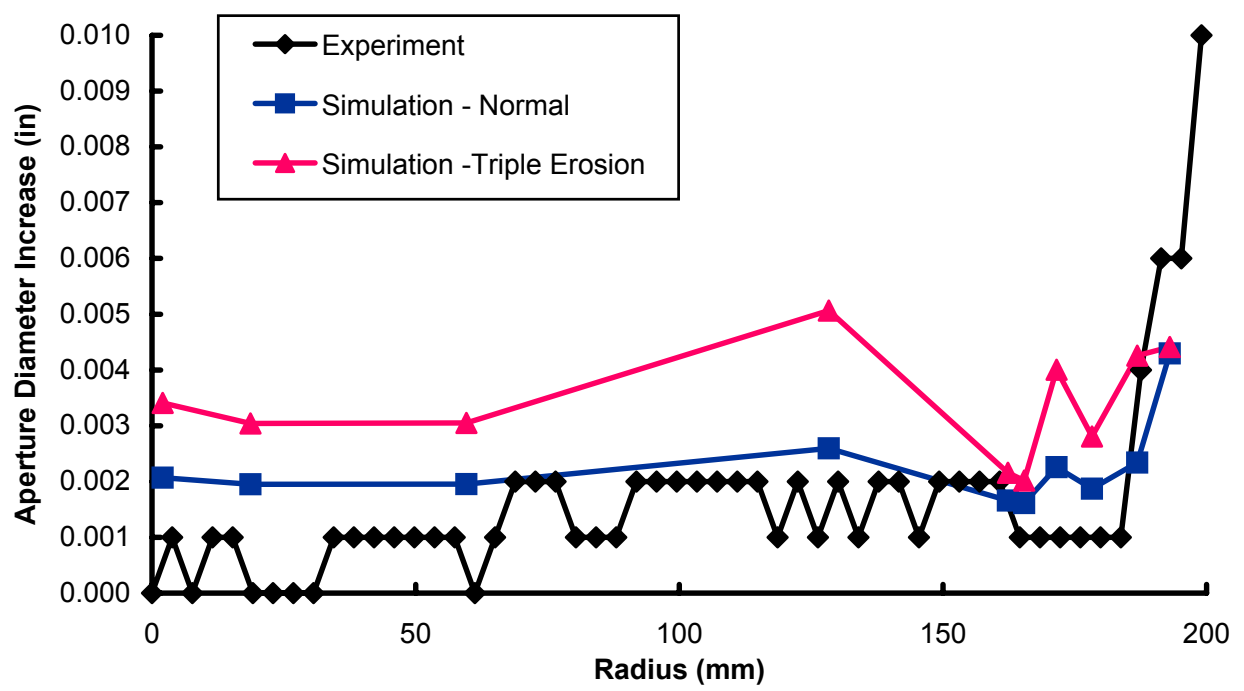

Figure 5. Aperture diameter increase due to erosion after 2000 hours of thruster operation. The pin-gauge measured experimental data and the simulation results are shown. Results for tripled erosion rates are plotted as well. Cross-over of the beamlet in the low-current apertures at high radii causes the large amount of erosion seen in these apertures.

Figure 5 shows the increase in accelerator grid aperture diameters following 2000 hours of thruster operation. Experimentally measured pin gauge results and simulation results are plotted. Also shown are the aperture diameters assuming an erosion rate three times higher than is simulated, representing a first 
order estimate of the erosion from the simulation for an accelerator grid current similar to the measured value. The erosion in the simulations compares well to the experimental data, as it is generally higher than the measured value by approximately 0.001 inches or less. There is more error for apertures near the thruster edge because these apertures experience direct impingement due to beamlet cross-over, as well as non-circular erosion due to misalignment of the screen and accelerator grid apertures. The axisymmetric simulation is unable to accurately model this type of erosion.

The normal erosion matches the experimental data well, while the tripled erosion rate data is much higher than the experimentally measured values. This indicates that the barrel erosion rate is approximately correct, and thus the current collected on the barrel can be assumed to be correct as well. If the simulation is at fault for the deficient accelerator grid current, this current must be collected on the downstream face of the grid.

Consider the centerline aperture only. The experimental current collected from this aperture should be approximately $0.61 \mu \mathrm{A}$. The simulated barrel current for this aperture is $0.081 \mu \mathrm{A}$, leaving $0.53 \mu \mathrm{A}$ of current to be collected on the downstream face. Assuming that all ions impact the grid surface at $230 \mathrm{eV}$ and normal incidence, this amount of current will erode approximately $1.4 \mathrm{mg}$ of grid material over 2000 hours. An estimate of the eroded mass seen in the 2000 hour wear test of the NEXT thruster may be made based on profilometer measurements. ${ }^{4}$ This estimate gives $0.47 \mathrm{mg}$ of eroded grid material around a single aperture, one third the value calculated based on the measured current. The simulation gives $0.25 \mathrm{mg}$ of eroded material after 2000 hours of erosion, or half the estimated value. These erosion values are summarized in Table 2 .

Table 2. Erosion estimates and calculations for the centerline aperture of the NEXT thruster after 2000 hours of operation.

\begin{tabular}{c|c|c}
$\begin{array}{c}\text { Inferred from measured } \\
\text { accelerator grid current }\end{array}$ & $\begin{array}{c}\text { Inferred from } \\
\text { profilometry data }\end{array}$ & Simulation \\
\hline $1.4 \mathrm{mg}$ & $0.47 \mathrm{mg}$ & $0.25 \mathrm{mg}$
\end{tabular}

The erosion inferred from the measured accelerator grid current given above, and the simulated erosion on the downstream face, do not take into account reduced erosion due to the pit and groove structures. As these form, eroded material is more likely to recombine on the wall of the pit or groove because the viewing angle of the sputtered material with respect to the grid walls is increased. Also, the angle of incidence of impacting ions will decrease as the walls of the pit or groove become steeper. However, it is not likely that this accounts for three times less erosion than the amount inferred from the measured accelerator grid current. The groove and pit structures are not deep after this amount of time, so any effect which might reduce erosion would be small. Also, there is some uncertainty in the sputter yields, but not enough for a factor of three difference. The simulation underestimates the amount of erosion somewhat, but there is still a large discrepancy between the erosion values inferred from the measurements of accelerator grid current and profilometry. This suggests that some portion of the measured accelerator grid current originates from a location other than around the ion optics apertures.

A 3-dimensional simulation of the downstream accelerator grid face erosion is also required for further study, as the present model cannot reproduce the pit and groove structures. If the downstream face erosion in a 3-D simulation matches the experimental data, and the accelerator grid current is still deficient, then the experimental current is being collected at a point that is not intended to be simulated. 3-D simulations of downstream face erosion have been performed by Farnell et al. ${ }^{6}$ and Wang et al. ${ }^{20}$ but it is unknown if the accelerator grid current was deficient in these cases.

\section{Electron Backstreaming Study}

One of the primary failure modes of an ion thruster is loss of performance due to electron backstreaming. This occurs when the accelerator grid aperture diameter increases due to erosion, such that there is no longer a retarding potential keeping plume electrons from being accelerated into the discharge chamber. This form of engine failure may be mitigated by increasing the potential on the accelerator grid; however, this also has the effect of accelerating the erosion, as impacting ions will have a higher energy. 
The onset of electron backstreaming occurs when the minimum centerline potential in the ion optics rises to a point such that electrons are able to backstream. The centerline will always have the highest potential, as it is furthest from the accelerator grid, and also because the ion density is generally highest on the centerline.

The electron backstreaming limit in the NEXT ion engine has previously been measured experimentally. $^{2,3}$ The backstreaming limit for a given ion optics geometry is measured by increasing the accelerator grid potential until the measured beam current increases by $0.1 \mathrm{~mA}$. This is approximately $3.36 \cdot 10^{-9} \mathrm{~A}$ of electron current for a single aperture. Apertures near the centerline of the thruster will generally produce backstreaming before the other apertures, because the higher ion density and larger aperture diameters result in a higher minimum centerline potential. For these reasons, the centerline aperture is simulated in the following cases.

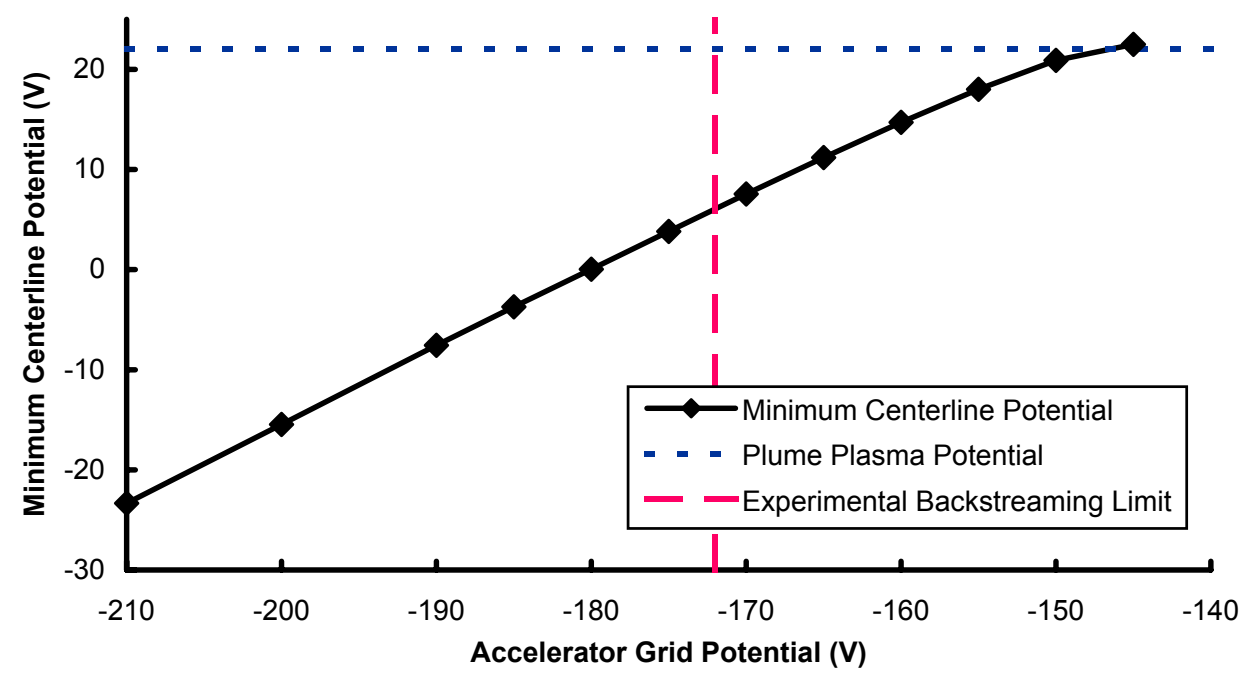

Figure 6. Minimum centerline potential as a function of accelerator grid potential. The downstream plasma potential and experimental backstreaming potential are also shown.

Figure 6 shows the simulated minimum centerline potential as a function of accelerator grid potential. Also shown is the plume plasma potential of $22 \mathrm{~V}$, and the experimentally measured backstreaming potential of $-172 \mathrm{~V}$. The plot shows that the minimum centerline potential at the experimentally measured backstreaming limit is about 7.5 V. For the simulation, this is then the point at which electron backstreaming is assumed to occur.

For the simulated plume plasma potential of $22 \mathrm{~V}$ and electron temperature of $1 \mathrm{eV}$, the single-aperture electron backflow current at this point is estimated to be $4 \cdot 10^{-13} \mathrm{~A}$, several orders of magnitude lower than the $3.36 \cdot 10^{-9} \mathrm{~A}$ limit used experimentally. However, experimental measurements ${ }^{4}$ indicate that the plume plasma potential is approximately $16 \mathrm{~V}$, not $22 \mathrm{~V}$. If a $16 \mathrm{~V}$ plume potential is assumed, then the electron backflow current will be $8.8 \cdot 10^{-11} \mathrm{~A}$, somewhat closer to the experimental limiting value, although still two orders of magnitude too low. The electron current is also very sensitive to the electron temperature. ${ }^{13}$ An increase to $1.5 \mathrm{eV}$ is sufficient to reach the experimental electron current limit for a $16 \mathrm{~V}$ plume potential. The minimum centerline potential is not affected strongly by the electron population properties, so the $7.5 \mathrm{~V}$ potential is used as the indicator of electron backstreaming, rather than the electron backflow current itself.

Although the electron backstreaming limit is found experimentally by varying the accelerator grid potential, the thruster will actually fail when the accelerator grid aperture diameters increase enough such that backstreaming occurs. For simulations where the aperture is eroded until thruster failure, it is more useful to know at what aperture diameter electron backstreaming will occur.

In order to determine the necessary aperture diameter to allow electron backstreaming, several simulations are performed with a gradually increasing diameter while the grid potential is held constant at $-210 \mathrm{~V}$. In each case, one layer of cells is removed from the accelerator grid barrel. Figure 7 shows the resulting minimum centerline potential as a function of aperture diameter. The diameters here are normalized by the nominal aperture diameter. Electron backstreaming occurs when the aperture enlarges by approximately $35 \%$.

This method of aperture enlargement does not account for reduction in the thickness of the grid, and 


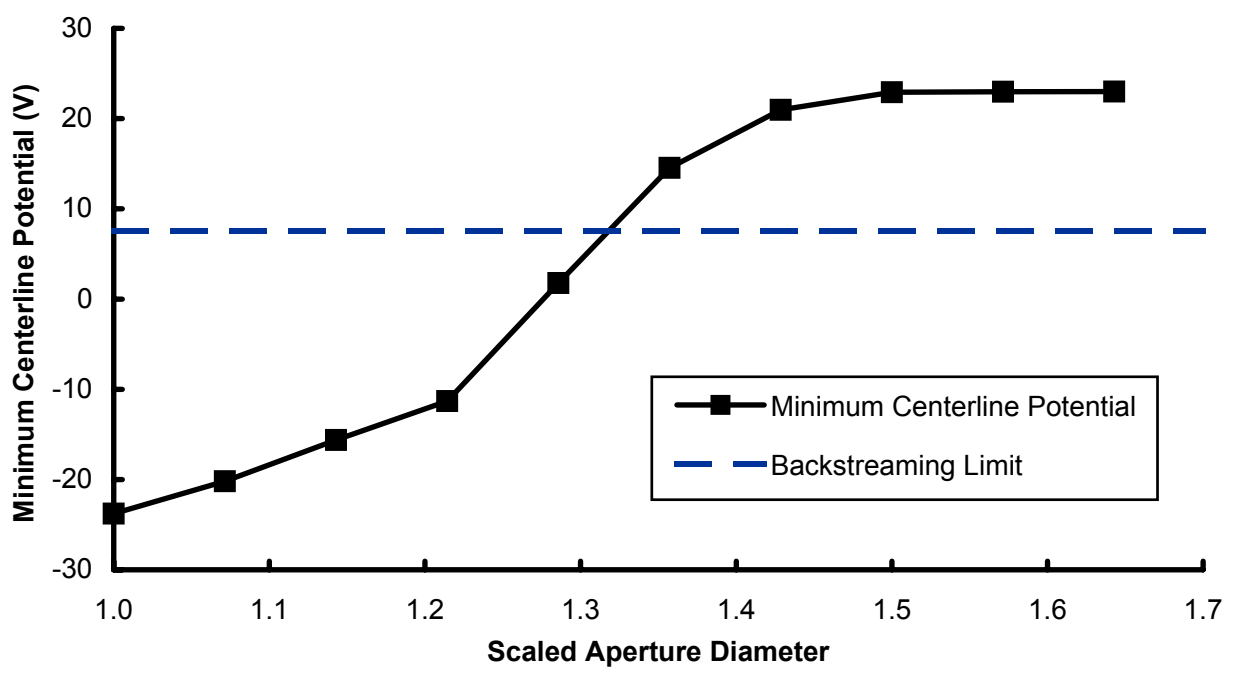

Figure 7. Minimum centerline potential as a function of accelerator grid aperture diameter. Also shown is the 7.5 $\mathrm{V}$ potential at which electron backstreaming will occur. The accelerator grid potential is $-210 \mathrm{~V}$ for these cases.

also assumes that the aperture diameter is increasing uniformly. A thinner grid will allow backstreaming to occur sooner, and a non-uniform erosion pattern may do this as well.

\section{Thruster Life Modeling Results}

Thruster failure due to electron backstreaming can be estimated by the model in several ways. A single simulation gives results for the erosion rate in each accelerator grid cell. These erosion rates can be used to erode the ion optics until the aperture is large enough to allow electron backstreaming. Another method is to use the erosion rates from the nominal geometry to erode over a set amount of time. Then a new simulation is run with the eroded geometry, and the erosion rates from the second simulation are used to erode the grid further. This process is continued until electron backstreaming occurs. Finally, a dynamic erosion algorithm may be used to erode the ion optics during a single simulation.

The axisymmetric domain of the model does not allow for accurate simulation of erosion on the downstream face of the ion optics. The hexagonal arrangement of the apertures in the optics creates a "pit-andgroove" pattern, where pits form between three adjacent apertures and grooves form between two adjacent apertures. The simulation is unable to model either of these structures. Thus, estimation of the life of the ion optics before they incur structural failure is not possible with the current model.

\section{A. Multiple and Single Simulation Results}

The thruster life is first estimated using multiple simulations with static geometry. In each case, the cusped NEXT ion optics wear test geometry is simulated at the $3.52 \mathrm{~A}, 1800 \mathrm{~V}$ operating point. The peak beamlet current of approximately $0.168 \mathrm{~mA}$ is modeled and the accelerator grid potential is fixed at $-210 \mathrm{~V}$. Five simulations are performed in total, with 10,000 hours of erosion being simulated at each step. The initial geometry is simulated first, which gives erosion rates on the accelerator grid barrel. These erosion rates are applied to the geometry to erode it for 10,000 hours. The $10 \mathrm{k}$-hr erosion case is then simulated to give updated erosion rates, which are used to erode to $20 \mathrm{k}$-hr. This is done until electron backstreaming occurs at after approximately $40 \mathrm{k}-\mathrm{hr}$ of erosion. At each step, the entire flow field is updated, not just the grid geometry. This ensures that the evolution of the erosion pattern is as accurate as possible.

For each column of simulated optics cells, the erosion rate is applied to the cell with the lowest radius. Erosion then occurs in a strictly radial fashion, and downstream face erosion is not be accounted for. Also, because the erosion simulated on the downstream corner of the grid includes erosion of the downstream face, the erosion for that cell is always set to be the same as for the cell directly next to it. 


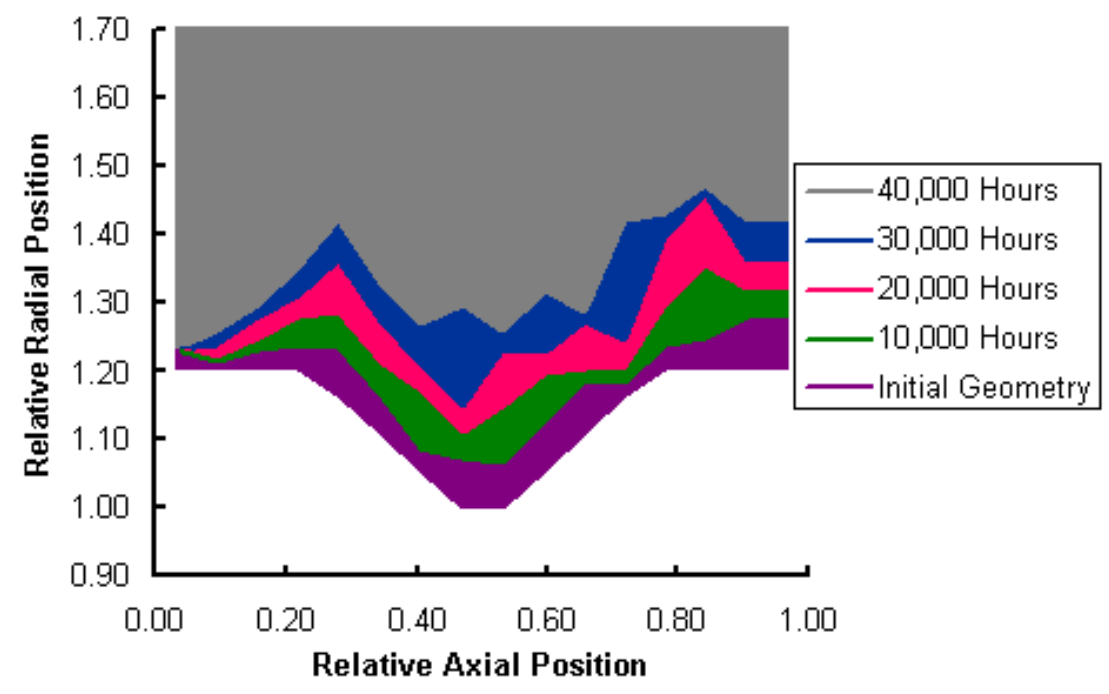

Figure 8. Geometry of the accelerator grid barrel as a function of erosion time. The axial position is normalized by the grid thickness, with its origin at the grid upstream surface. The radial position is normalized by the initial aperture radius - the origin is on the domain centerline.

The ion optics geometry at each simulation step is shown in Figure 8. Very little erosion occurs on the upstream surface of the grid, so these points do not vary greatly over the life of the thruster. The erosion rate then increases towards the center of the grid, creating a pit in front of the grid midpoint. This pit is caused by high-energy CEX ions created in the inter-grid region. Erosion in the center region is average, followed by another region of high erosion. The erosion is higher on the downstream half of the grid due to CEX ions created near the accelerator grid aperture and in the downstream region.

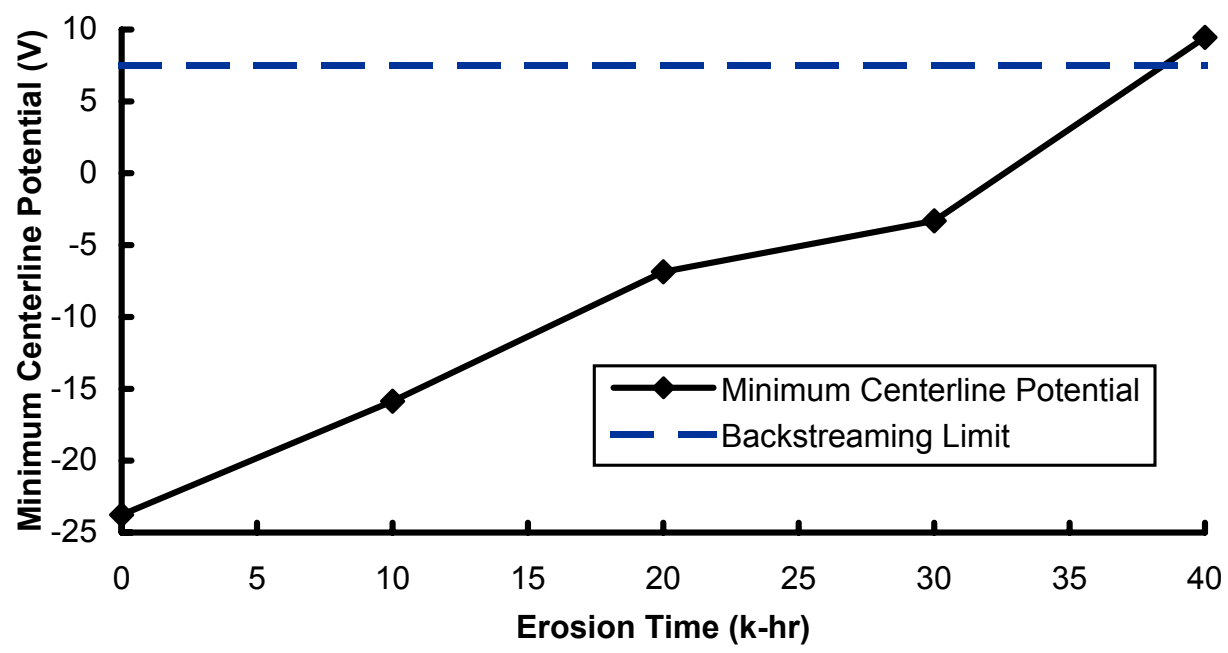

Figure 9. Minimum centerline potential as a function of erosion time. The backstreaming limit is also shown. The potential varies linearly with time, indicating that backstreaming may be predicted based solely on initial erosion rates.

The minimum centerline potential as a function of erosion time is plotted in Figure 9. As the plot shows, the electron backstreaming limit is reached shortly before $40 \mathrm{k}$-hr of erosion. The increase in potential is approximately linear with time as well, which indicates that the backstreaming behavior can be predicted easily given initial erosion rates.

The life of the thruster may also be estimated by eroding the accelerator grid barrel using only erosion rates simulated at the initial geometry. The results plotted in Figure 9 indicate that this method will be 
effective. Indeed, electron backstreaming is found to occur in this case after approximately $40 \mathrm{k}$-hr of thruster operation, the same as seen in the multiple simulation case. Figure 10 plots the final geometries for both single-simulation and multiple-simulation results. The geometries are very similar, although the multiplesimulation result is smoother, due to the erosion being re-distributed as some parts of the grid are worn away.

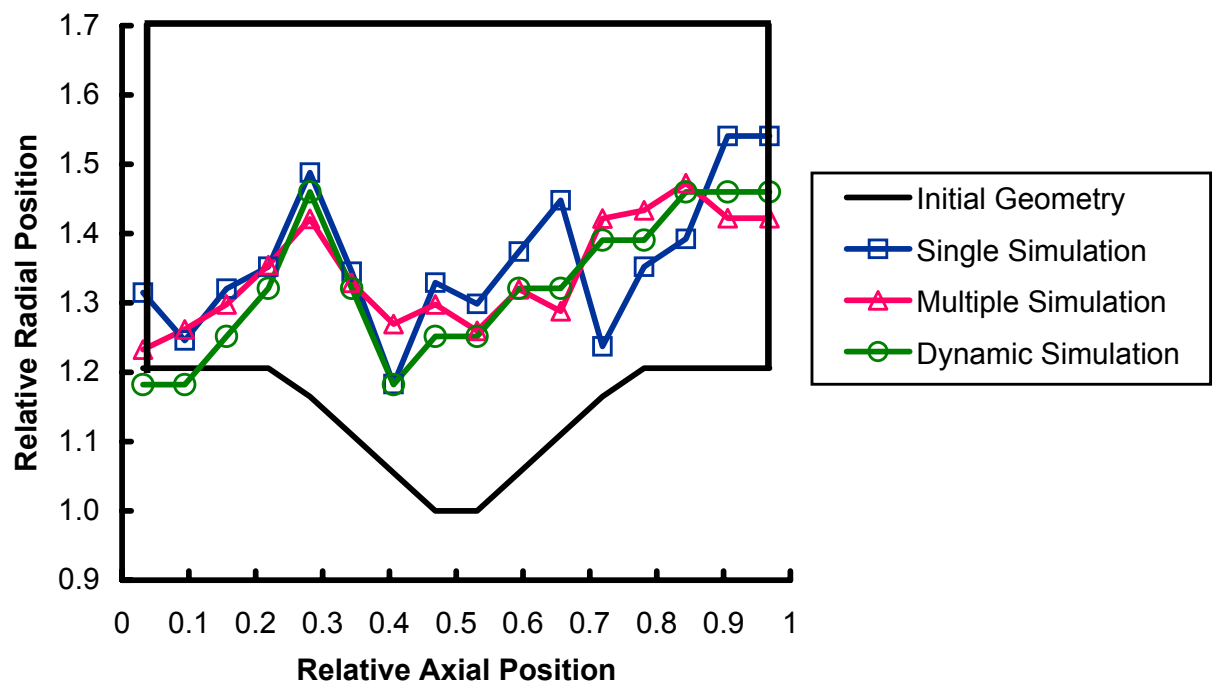

Figure 10. End-of-life accelerator grid barrel geometry for single, multiple, and dynamic simulations. The single and multiple simulation final geometries are both reached after approximately 40,000 hours of erosion. The multiple-simulation case is generally smoother because erosion is redistributed as different parts of the grid are eroded. The final dynamic erosion geometry is reached after 45,000 hours of erosion.

\section{B. Dynamic Erosion Results}

Finally, erosion is modeled using dynamic erosion of the ion optics. In this case, the simulation begins with the initial geometry, and initializes the flow using this geometry. Once the flow is initialized, dynamic erosion is enabled. Whenever an ion impacts on an optics surface, the number of eroded molybdenum atoms is calculated. These atoms are removed from the optics cell, and if all of the atoms are removed from a cell, that cell is no longer considered an ion optics cell. Re-deposition of sputtered grid material is not simulated here.

The simulation time-step is on the order of $1 \cdot 10^{-10}$ seconds, and the dynamic erosion is performed for 100,000 iterations, giving a total simulated time on the order of 0.01 milliseconds. To allow simulation of thruster life, the number of atoms in an optics cell must be scaled. This scaling factor is simply the amount of time the simulation actually models divided by the thruster erosion time. In this simulation, the thruster erosion time is set to 50,000 hours, so the scaling factor is approximately $3 \cdot 10^{-13}$.

For a better comparison to the multiple and single simulation results, the dynamic erosion algorithm is restricted such that downstream face erosion does not occur or is mitigated. This is accomplished by not eroding material when an ion impacts on the downstream face of the grid. However, this still allows impacts on the downstream side of any eroded geometry or the cusps.

This method produces results very close to the multiple and single aperture simulation erosion. The eroded grid geometry and potential field after 15, 30, and $45 \mathrm{k}$-hr of erosion are plotted in Figure 11. As before, electron backstreaming is assumed to occur when the $7.5 \mathrm{~V}$ contour is connected between the up and downstream regions. In this case, backstreaming occurs after 45,000 hours of erosion. Note that the downstream half of the grid is systematically chamfered by CEX ions from the downstream region. Although erosion is not allowed by these ions on the actual downstream face, they do contribute to erosion in other areas.

As shown by Figure 10, the dynamic erosion geometry at which backstreaming occurs is very similar to the previous results. One difference is that this result is reached after approximately 45,000 hours of erosion, rather than the 40,000 hours needed in the multiple and single simulation cases. This difference most likely 

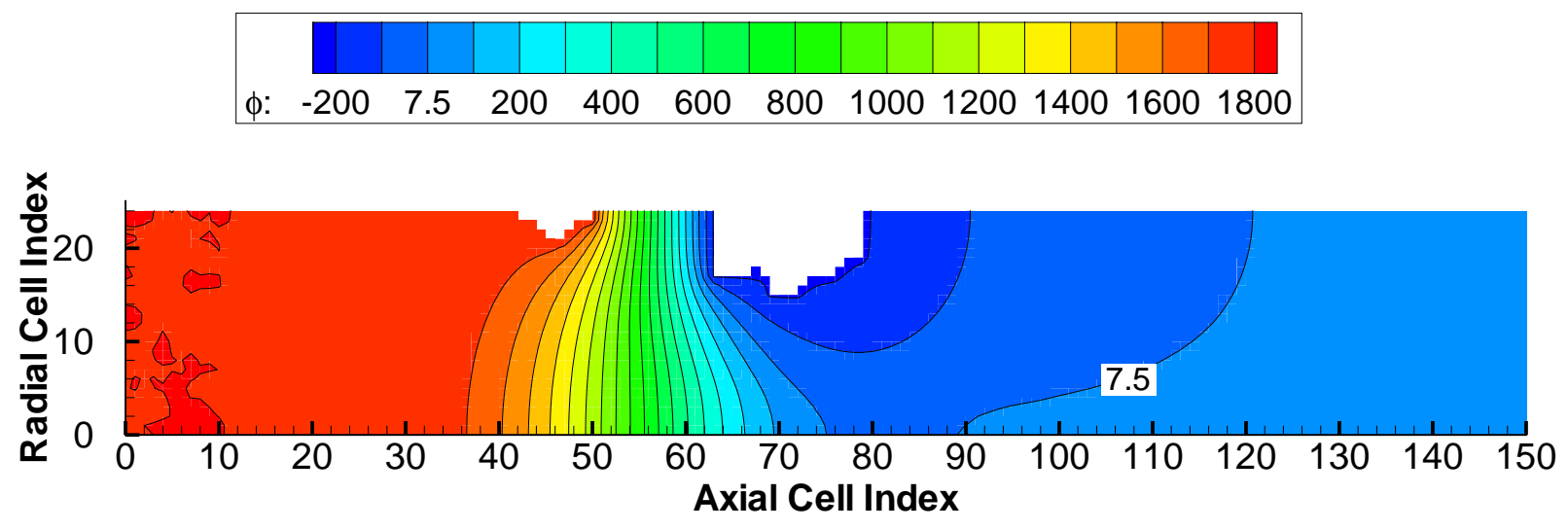

(a) 15,000 Hours of erosion

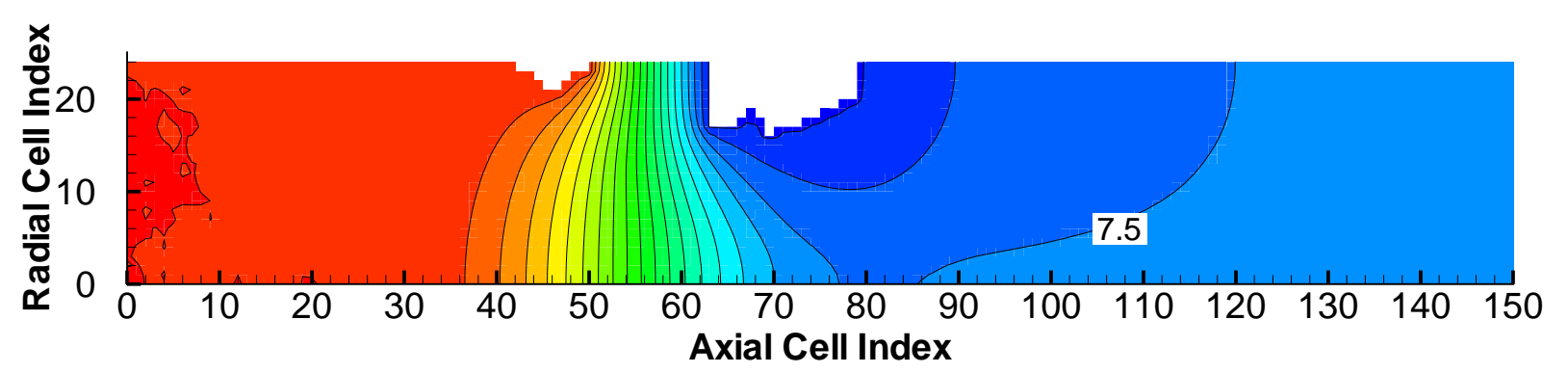

(b) 30,000 Hours of erosion

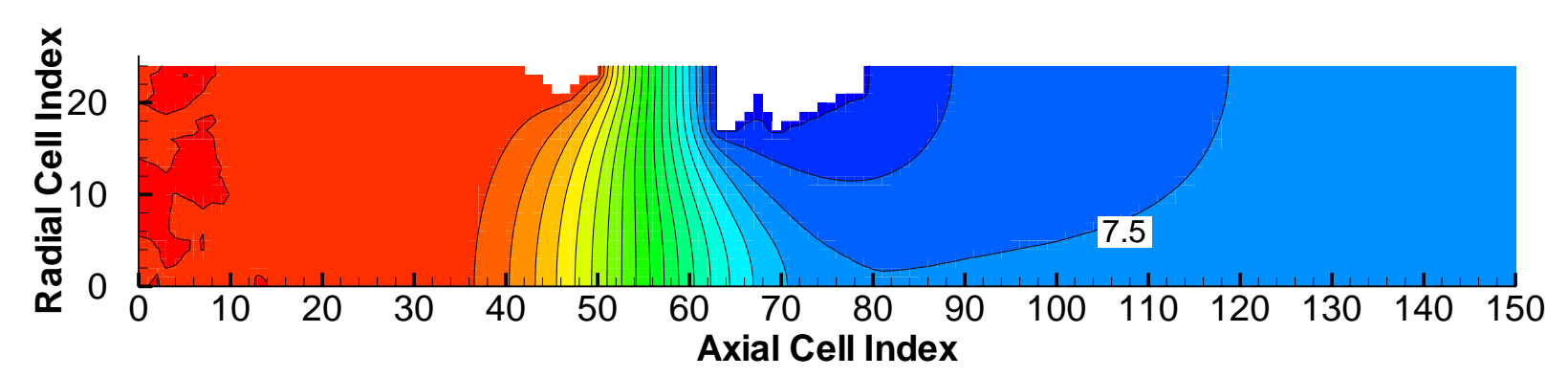

(c) 45,000 Hours of erosion

Figure 11. Potential field contours as the accelerator grid is eroded dynamically, without downstream face erosion. Dimensions are in volts. The $7.5 \mathrm{~V}$ contour is labelled- when the downstream contour connects with the upstream contour, electron backstreaming will occur. After approximately 45,000 hours of erosion, backstreaming begins. 
occurs because the geometry simulated in the dynamic erosion case depends on the mesh of the domain. Thus, as the first two points in Figure 10 show, the dynamic erosion case must erode more material in some areas. In the other types of simulations, the true geometry is eroded using the simulated erosion rates. Also, the potential field for the dynamic erosion case is a snapshot- it is the potential for only one iteration. The other cases are able to average the potential over many iterations to find the minimum centerline potential. Thus, the potential in the dynamic case may involve some statistical fluctuations.

\section{Summary}

The erosion predictions given here for the single and multiple simulation cases predict thruster failure after approximately 40,000 hours of thruster operation at the maximum operating point. This corresponds to $845 \mathrm{~kg}$ of propellent throughput. This is likely a very optimistic prediction given that downstream face erosion is ignored. However, it is apparent that erosion rates from a single simulation are sufficient to predict electron backstreaming behavior.

This model has been used previously to predict electron backstreaming. In previous simulations, ${ }^{11}$ the backstreaming limit was reached after approximately the same thruster operating time. Results for a 3-D model ${ }^{6}$ predict thruster failure much sooner, after a propellant throughput of $625 \mathrm{~kg}$. However, this estimate is for an accelerator grid potential of $-250 \mathrm{~V}$, and is due to structural failure, not electron backstreaming. These results define thruster failure due to structural failure as the point at which $50 \%$ of the grid material has been eroded. At an accelerator grid potential of $-200 \mathrm{~V}$, the estimate increases to $805 \mathrm{~kg}$ of throughput, much closer to the result given above, although end-of-life is again due to structural failure in this case. The rapid erosion of the downstream face in the dynamic erosion simulation supports this prediction. Predictions based on experimental data give failure after $750 \mathrm{~kg}$ of throughput, ${ }^{3}$ also due to structural failure. In this case, structural failure is defined as the point at which the grooves will erode through the grid.

\section{Conclusion}

PIC simulation of several ion optics apertures at different radii on the thruster surface provides a more accurate picture of thruster performance than simply using results from a single simulation. The model described here is capable of accurately simulating performance of the NEXT ion engine. Accelerator grid barrel erosion is also modeled correctly, but discrepancies between the current collected and the downstream face erosion of the grid indicate that the experimentally measured current may be too high.

Three methods can be used to estimate the life of the thruster before the onset of electron backstreaming due to aperture enlargement. The use of a single simulation, multiple simulations, and dynamic erosion all predict that the NEXT ion engine will encounter electron backstreaming after approximately 40,000 hours of operation, or $845 \mathrm{~kg}$ of xenon propellant throughput.

\section{Acknowledgements}

Support for this work is provided in part by NASA Glenn Research Center Grant NAG3-2497 with Jon Van Noord as the technical monitor. Support is also provided by a Michigan Space Grant Consortium Graduate Student Fellowship.

\section{References}

\footnotetext{
${ }^{1}$ Brophy, J. R., Brinza, D. E., Polk, J. E., Henry, M. D., and Sengupta, A., "The DS1 Hyper-Extended Mission," AIAA 2002-3673, 38th AIAA/ASME/SAE/ASEE Joint Propulsion Conference, Indianapolis, IN, July 2002.

${ }^{2}$ Soulas, G. C., Haag, T. W., and Patterson, M. J., "Performance Evaluation of $40 \mathrm{~cm}$ Ion Optics for the NEXT Ion Engine," AIAA 2002-3834, 38th AIAA/ASME/SAE/ASEE Joint Propulsion Conference, Indianapolis, IN, July 2002.

${ }^{3}$ Soulas, G. C., Kamhawi, H., Patterson, M. J., Briton, M. A., and Frandina, M. M., "NEXT Ion Engine 2000 Hour Wear Test Results," AIAA 2004-3791, 40th AIAA/ASME/SAE/ASEE Joint Propulsion Conference, Fort Lauderdale, FL, July 2004.

${ }^{4}$ Kamhawi, H., Soulas, G. C., Patterson, M. J., and Frandina, M. M., "NEXT Ion Engine 2000 Hour Wear Test Plume and Erosion Results," AIAA 2004-3792, 40th AIAA/ASME/SAE/ASEE Joint Propulsion Conference, Fort Lauderdale, FL, July 2004.

${ }^{5}$ Malone, S. P. and Soulas, G. C., "Computational Ion Optics Design Evaluations," AIAA 2004-3784, 40th AIAA/ASME/SAE/ASEE Joint Propulsion Conference, Fort Lauderdale, FL, July 2004.
} 
${ }^{6}$ Farnell, C. C., Williams, J. D., and Wilbur, P. J., "NEXT Ion Optics Simulation Via ffx," AIAA 2003-4869, 39th AIAA/ASME/SAE/ASEE Joint Propulsion Conference, Huntsville, AL, July 2003.

${ }^{7}$ Kafafy, R. and Wang, J., "Whole Ion Optics Simulations of a Subscale Gridlet Using a Hybrid-Grid IFE-PIC Code," AIAA 2004-3783, 40th AIAA/ASME/SAE/ASEE Joint Propulsion Conference, Fort Lauderdale, FL, July 2004.

${ }^{8}$ Okawa, Y., Hayakawa, Y., and Kitamura, S., "Three-Dimensional Divergence Characteristics of Ion Beamlets in an Ion Thruster," AIAA 2004-3785, 40th AIAA/ASME/SAE/ASEE Joint Propulsion Conference, Fort Lauderdale, FL, July 2004.

${ }^{9}$ Crofton, M. W. and Boyd, I. D., "The Origins of Accelerator Grid Current: Analysis of T5-Grid Test Results," AIAA 99-2443, 35th AIAA/ASME/SAE/ASEE Joint Propulsion Conference, Los Angeles, CA, June 1999.

${ }^{10}$ Emhoff, J. W. and Boyd, I. D., "A Numerical Study of Neutralization and Sputtering Processes in the NSTAR Thruster," AIAA 2002-4259, 38th AIAA/ASME/SAE/ASEE Joint Propulsion Conference, Indianapolis, IN, July 2002.

${ }^{11}$ Emhoff, J. W. and Boyd, I. D., "Grid Erosion Modeling of the NEXT Ion Thruster Optics," AIAA 2003-4869, 39th AIAA/ASME/SAE/ASEE Joint Propulsion Conference, Huntsville, AL, July 2003.

${ }^{12}$ Emhoff, J. W. and Boyd, I. D., "Progress in NEXT Ion Optics Modeling," AIAA 2004-3786, 40th AIAA/ASME/SAE/ASEE Joint Propulsion Conference, Fort Lauderdale, FL, July 2004.

${ }^{13}$ Emhoff, J. W., Simulation of Ion Optics Using Particle-In-Cell and Treecode Methods, Ph.D. thesis, University of Michigan, 2005.

${ }^{14}$ Birdsall, C. K. and Langdon, A. B., Plasma Physics Via Computer Simulation, Adam Hilger Press, 1991.

${ }^{15}$ Bird, G. A., Molecular Gas Dynamics and the Direct Simulation of Gas Flows, Oxford University Press, 1994.

${ }^{16}$ Polk, J. E., Moore, N. R., Newlin, L. E., Brophy, J. R., and Ebbeler, D. H., "Probabilistic Analysis of Ion Engine Accelerator Grid Life," IEPC-93-176, 23rd International Electric Propulsion Conference, Seattle, WA, September 1993.

${ }^{17}$ Polk, J. E., "Personal Communication," September 2002.

${ }^{18}$ Soulas, G. C., Domonkos, M. T., Kamhawi, H., and Patterson, M. J., "Status of the NEXT Ion Engine Wear Test," AIAA 2003-4863, 39th AIAA/ASME/SAE/ASEE Joint Propulsion Conference, Huntsville, AL, July 2003.

${ }^{19}$ Dalgarno, A., McDowell, M. R. C., and Williams, A., "The Mobilities of Ions in Unlike Gases," Royal Society of London Philosophical Transactions Series A, Vol. 250, 1958, pp. 411-425.

${ }^{20}$ Wang, J., Polk, J., Brophy, J., and Katz, I., "Three-Dimensional Particle Simulations of Ion-Optics Plasma Flow and Grid Erosion," AIAA Journal of Propulsion and Power, Vol. 19, No. 6, November-December 2003, pp. 1192-1199. 